consultants for assistance in the review and implementation of our framework; provided initial racial equity training for our staff and board; created a blog series highlighting the gun violence epidemic in communities disproportionately affected by gun violence; applied findings in policy development work with researchers.

Conclusions A racial equity framework will help mitigate potentially harmful inequities in GVP, and instead will promote social justice.

Significance/Contributions Equity is an established and necessary component of public health that has received little attention in GVP. The Ed Fund's self-evaluation, training, and development/application of a racial equity framework for GVP provides guidance and context for researchers, advocates, partner organizations, and policymakers to consider and advance in their own work. This framework is modifiable for a variety of violence and injury topics.

\section{CHARACTERISTICS OF HOSPITALS THAT CARE FOR PATIENTS WITH FIREARM INJURIES: EVIDENCE FROM THE NATIONWIDE EMERGNCY DEPARTMENT SAMPLE}

Elinore Kaufman, Kit Delgado. University of Pennsy/vania

10.1136/injuryprev-2020-savir.132

Statement of Purpose Firearm injury is a public health crisis in the United States, but little is known about the distribution of firearm injury care among health systems. We examined national estimates of ED visits and hospital admissions to describe the hospitals that care for patients with firearm injuries. This research aims to identify areas of focus for practice improvement and hospital-based violence prevention.

Methods Data was collected from the Nationwide ED Sample (NEDS) for 2016. The NEDS is a $20 \%$ stratified sample of all U.S. EDs weighted to provide national estimates. We identified firearm-related injuries using ICD-10 external cause-ofinjury codes. We described the distribution of firearm injuries among hospitals and characterized hospitals that treat an average of 2 firearm injuries per week (100/year) according to hospital size, ownership, region, teaching status, urban-rural location, and region.

Results Of the 953 hospitals in the NEDS, 818 hospitals weighted to represent 3,892 hospitals nationally treated a total of 97,608 firearm injuries in 2016. The median firearm injuries treated was 8 (interquartile range 3, 19). The 226 hospitals $(5.8 \%)$ that treated $\geq 100$ firearm injuries cared for $47.4 \%$ of all firearm injuries, $69.0 \%$ of 26,812 admissions, $60.3 \%$ of 12,775 severely injured patients, and $58.3 \%$ of 7,626 deaths. Eighty-two percent were Level I or II trauma centers and 91\% were metropolitan, teaching hospitals, with 57\% located in major urban centers. Forty-six percent were in the South, $37 \%$ in the West, $29 \%$ in the Midwest, and $8 \%$ in the Northeast.

Conclusions Most U.S. hospitals care for firearm injuries, but care is concentrated in a small minority of hospitals, primarily in metropolitan trauma centers in the South and West.

Significance and Contributions to Injury and Violence Prevention Science Hospitals treating substantial numbers of gunshot wound patients may be the most appropriate sites for hospital-based violence intervention programs.

\section{CREDIT SCORES AS A NOVEL MEASUREMENT TOOL TO EXAMINE VIOLENCE INEQUITIES IN PHILADELPHIA, PA}

Mudia Uzzi, Lorraine Dean. Johns Hopkins School of Public Health

10.1136/injuryprev-2020-savir.133

Statement of Purpose Neighborhood Violence is a major social and public health issue throughout the United States and specifically in urban areas. Credit scores are an emerging novel measurement tool to examine violence across communities. Area-level credit scores reflect elements of neighborhoods' economic structure that is not captured by traditional socioeconomic position measures.

Methods/Approach Our ecological cross-sectional study obtained Census Block Group (CBG) Equifax Vantage 3.0 credit score averages for all CBGs $(n=1,325)$ in Philadelphia, PA from 2013-2017. We joined credit score data with geocoded data from 78,184 individual violent crime incidents between 2013-2017 and across Philadelphia CBGs. We created five separate analytic datasets for each year of our study time period by combining credit score and violence CBG data with covariates from the US Census. We used Poisson regression to determine associations between CBG credit scores and CBG violent crime counts, after controlling for population density and percentages of home ownership, vacant houses, black residents, female-headed households, and poverty.

Results Across the five-year period, 27\%-32\% of CBGs had poor/fair credit score averages, 56-57\% had good, and 12\%$16 \%$ had excellent credit score averages. Moreover, violent crime counts per CBG across the five-year period ranged from 0-93 with a median of $8-10$ incidents per CBG. CBGs with good credit scores had $18 \%$ - 28\% less risk of violent crime compared to poor/fair credit CBGs. Furthermore, CBGs with excellent credit scores had a 36\%-46\% less risk of violent crime compared to poor/fair credit CBGs.

Conclusions Excellent and good credit score averages by CBG are associated with lower violent crime counts compared to CBGs with poor/fair averages across Philadelphia.

Significance and Contributions to Injury and Violence Prevention Science Credit scores have potential for improving the understanding of violence inequities and what contributes to violence, over and above traditional socioeconomic position measures.

\section{THE RISK OF WATCHING A SUNSET - EPIDEMIOLOGIC FEATURES OF A COHORT OF COASTAL CLIFF-RELATED TRAUMATIC INJURY}

Kathryn Schaffer. American Trauma Society; American Association of Surgical Trauma; Trauma Quality Improvement Program

\subsection{6/injuryprev-2020-savir.134}

Statement of Purpose California coastal cliffs are known for scenic views, natural beauty inviting the public to explore, but are also known for environmental risks. An increase of falls from cliffs with severe outcomes, combined with risky behaviors, was identified. A review describing cliff-related falls from two geographically different coastal regions was done.

Methods/Approach All trauma admissions 2010-2018 from two trauma centers (TC) was reviewed for unintentional falls at coastal cliffs. Study sites have coastline catchments with 